TRANSACTIONS OF THE

AMERICAN MATHEMATICAL SOCIETY

Volume 348, Number 5, May 1996

\title{
FUCHSIAN SUBGROUPS OF BIANCHI GROUPS
}

\author{
D. G. JAMES AND C. MACLACHLAN
}

\begin{abstract}
A maximal non-elementary Fuchsian subgroup of a Bianchi group $P S L\left(2, O_{d}\right)$ has an invariant circle or straight line under its linear fractional action on the complex plane, to which is associated a positive integer $D$, the discriminant, which, in turn, is an invariant of the wide commensurability class of the Fuchsian subgroup. In this paper, for all Bianchi groups, we classify the conjugacy classes of these maximal Fuchsian subgroups by determining the number with given discriminant.
\end{abstract}

\section{INTRODUCTION}

Let $O_{d}$ denote the ring of integers in the quadratic number field $\mathbf{Q}(\sqrt{ }-d)$ where $d$ is a square-free positive integer. The Bianchi groups are the groups $P S L\left(2, O_{d}\right)$. (For a treatment of their algebraic aspects, see [4] and the references there.) The Bianchi groups are discrete subgroups of $\operatorname{PSL}(2, \mathbf{C})$. The elements of $\operatorname{PSL}(2, \mathbf{C})$ act via linear fractional transformation on the extended complex plane, and hence, using the Poincaré extension, on upper half 3 -space. Upper half 3 -space, $\{(x, y, z) \in$ $\left.\mathbf{R}^{3} \mid z>0\right\}$, equipped with the metric $d s=z^{-1} \sqrt{ }\left(d x^{2}+d y^{2}+d z^{2}\right)$ is a model of hyperbolic space $\mathbf{H}^{3}$ and under the above action, $\operatorname{PSL}(2, \mathbf{C})=\operatorname{Isom}{ }^{+}\left(\mathbf{H}^{3}\right)$, the orientation-preserving subgroup of the full isometry group of $\mathbf{H}^{3}$. The quotients of $\mathbf{H}^{3}$ by the actions of the groups $P S L\left(2, O_{d}\right)$ are then hyperbolic orbifolds of finite hyperbolic volume - the Bianchi orbifolds.

Let $\Gamma$ be a Kleinian group i.e. a discrete subgroup of $P S L(2, \mathbf{C})$. Then a subgroup $F$ is a Fuchsian subgroup of $\Gamma$ if there is a circle or straight line $\mathcal{C}$ in C which is invariant under the action of $F$ and such that the two components of $\mathbf{C} \backslash \mathcal{C}$ are preserved by $F$. All the Fuchsian groups considered here will be nonelementary, so that the limit set of $F$ on $\mathcal{C}$ contains more than two points. The hemisphere or plane $\hat{\mathcal{C}}$ orthogonal to $\mathcal{C}$ in upper half 3 -space is then invariant under $F$ and when the quotient $\hat{\mathcal{C}} / F$ is compact or has finite volume in the restriction of the hyperbolic metric to $\hat{\mathcal{C}}$, then $F$ is said to be cocompact or have finite covolume respectively. Any maximal Fuchsian subgroup $F$ of $\Gamma$ will be the full stabiliser in $\Gamma$ of $\mathcal{C}$, i.e. $F=\operatorname{Stab}(\mathcal{C}, \Gamma)$.

The Bianchi groups are rich in Fuchsian subgroups, each $P S L\left(2, O_{d}\right)$ having infinitely many commensurability classes of maximal Fuchsian subgroups [7]. Finiteness results in terms of suitable parameters for the conjugacy classes of maximal

Received by the editors November 11, 1994 and, in revised form, July 6, 1995.

1991 Mathematics Subject Classification. Primary 11F06; Secondary 11E08, 11E12, 20H10.

The first author partially supported by NSA and NSF grants.

Both authors would like to thank the Mathematics Department of the University of Auckland for its hospitality. 
Fuchsian subgroups in $P S L\left(2, O_{d}\right)$ have been obtained [8]. For those values of $d$ such that $d=1,2$ or $d=p$, a prime $\equiv 3(\bmod 4)$, there is a complete classification of these conjugacy classes [12]. This has been extended to include those values of $d$ for which the ideal class group of $\mathbf{Q}(\sqrt{ }-d)$ has no invariant divisible by 4 [13]. A complete analysis of the structure of the maximal Fuchsian subgroups in the case $d=1$ was also obtained in [8]. See also [2].

Each circle $\mathcal{C}$, which is stabilised by a non-elementary Fuchsian group $F$ of $P S L\left(2, O_{d}\right)$ determines a positive integer $D$, the discriminant of the circle, which is an invariant of the conjugacy class of $F$ (see $\S 3$ ). In this paper, for all square-free $d$, we completely determine the classification of the conjugacy classes of maximal Fuchsian subgroups by determining the number $n_{d}(D)$ of these with discriminant $D$ (see Theorem 5.7).

As in [8], the method is to convert the problem into one of determining primitive representations of rational integers by forms defined over $\mathbf{Q}$, modulo the action of groups commensurable with the unit group of this form. This was done for the case $d=1$ in [8] and here we carry out this conversion for general values of $d$. To pass from the groups $P S L\left(2, O_{d}\right)$ to groups of units of related quadratic forms is a special case of a principle applying to arithmetic Kleinian groups which contain non-elementary Fuchsian subgroups [9].

In all cases, the groups $\operatorname{PSL}\left(2, O_{d}\right)$ carry over to the spinorial groups $O^{\prime}(L)$ for a suitable lattice $L$ in the relevant quadratic space (see $\S 2$ ). Classifying the conjugacy classes of maximal non-elementary Fuchsian subgroups in $\operatorname{PSL}\left(2, O_{d}\right)$, then reduces, in essence, to counting orbits of elements of $L$ under the action of $O^{\prime}(L)$ (see $\S 3$ ). Using a local-global theorem similar to one proved in [6], the enumeration reduces to counting orbits in lattices over $p$-adic integers modulo the action of the local spinor groups of these lattices (see Theorem 4.1). Counting techniques in lattices over local rings are then used to complete the calculations (see $\S 5)$.

\section{BIANCHI GROUPS AND QUADRATIC FORMS}

In this section, the precise relationship between the Bianchi groups and the unit groups of the related integral forms is established.

Let $A$ denote the quaternion algebra $M(2, \mathbf{Q}(\sqrt{ }-d))$ with standard basis $1, i, j, i j$ where

$$
i=\left(\begin{array}{cc}
0 & 1 \\
-1 & 0
\end{array}\right), \quad j=\left(\begin{array}{cc}
1 & 0 \\
0 & -1
\end{array}\right) .
$$

Then the order $M\left(2, O_{d}\right)=O_{d}[1, i, 1 / 2(1+j), 1 / 2(i+i j)]$.

Now $A$ admits a conjugate-linear involution $\tau$ defined by

$$
\tau\left(a_{0}+a_{1} i+a_{2} j+a_{3} i j\right)=\overline{a_{0}}-\overline{a_{1}} i-\overline{a_{2}} j-\overline{a_{3}} i j
$$

whose fixed point set $V$ is a 4-dimensional space over $\mathbf{Q}$. With the restriction of the norm form, denoted by $n, V$ is a regular quadratic space with orthogonal group $O(V)$. Let $f$ denote the symmetric bilinear form

$$
f: V \times V \rightarrow \mathbf{Q}
$$

which is defined by

$$
f(v, w)=n(v+w)-n(v)-n(w)
$$


so that $f(v, v)=2 n(v)$. In $V$, fix a basis $\{r, s, t, u\}$ with $n(r)=1, n(s)=d, n(t)=$ $n(u)=0$ and $f(t, u)=d$ by choosing $r=1, s=(\sqrt{ }-d) j, t=(\sqrt{ }-d) / 2(-i+i j)$ and $u=(\sqrt{ }-d) / 2(i+i j)$.

Define the group $A_{\mathbf{Q}}^{*}$ by

$$
A_{\mathbf{Q}}^{*}=\left\{\beta \in A^{*} \mid \operatorname{det}(\beta) \in \mathbf{Q}^{*}\right\} .
$$

For $\beta \in A_{\mathbf{Q}}^{*}$ define $\phi_{\beta}: V \rightarrow V$ by

$$
\phi_{\beta}(v)=(\operatorname{det}(\beta))^{-1} \beta v \tau(\beta) .
$$

Then $\phi_{\beta} \in O(V)$ and we have a homomorphism

$$
\Phi: A_{\mathbf{Q}}^{*} \rightarrow O(V)
$$

defined by $\Phi(\beta)=\phi_{\beta}$.

We obtain a precise description of $\Phi$ in terms of the basis $\{r, s, t, u\}$ described above. Thus if $\beta=\left(\begin{array}{cc}x & y \\ z & w\end{array}\right)$, then $\Phi(\beta)$ is $(\operatorname{det}(\beta))^{-1}$ times the following $4 \times 4$ matrix:

$$
\left(\begin{array}{cccc}
\operatorname{Re}(x \bar{w}-y \bar{z}) & -d \operatorname{Im}(x \bar{w}+y \bar{z}) & -d \operatorname{Im}(x \bar{z}) & -d \operatorname{Im}(w \bar{y}) \\
\operatorname{Im}(x \bar{w}-y \bar{z}) & \operatorname{Re}(x \bar{w}+y \bar{z}) & \operatorname{Re}(x \bar{z}) & -\operatorname{Re}(w \bar{y}) \\
2 \operatorname{Im}(x \bar{y}) & 2 \operatorname{Re}(x \bar{y}) & x \bar{x} & -y \bar{y} \\
2 \operatorname{Im}(w \bar{z}) & -2 \operatorname{Re}(w \bar{z}) & -z \bar{z} & w \bar{w}
\end{array}\right) .
$$

The notation here is that, if $\alpha=a+b \sqrt{ }-d$ with $a, b \in \mathbf{Q}$, then $\operatorname{Re}(\alpha)=a$ and $\operatorname{Im}(\alpha)=b$.

We see immediately that the image of $A_{\mathbf{Q}}^{*}$ lies in $O(V)$ and that the kernel is $\mathrm{Q}^{*} I$. Let

$$
\theta: S O(V) \rightarrow \frac{\mathbf{Q}^{*}}{\mathbf{Q}^{* 2}}
$$

denote the spinor norm, with kernel $O^{\prime}(V)$, which is the commutator subgroup of $S O(V)$. Furthermore, it is the subgroup generated by all Eichler transformations [11].

Since $n(t)=0$ we can define for each $v \in V$ such that $f(t, v)=0$ the Eichler transformation $E(t, v)$ by

$$
E(t, v) w=w-f(t, w) v+f(v, w) t-n(v) f(t, w) t .
$$

Let $\beta \in S L(2, \mathbf{Q}(\sqrt{ }-d))$ have the form $\left(\begin{array}{ll}1 & \alpha \\ 0 & 1\end{array}\right)$ where $\alpha=a+b \sqrt{ }-d$. Then

$$
\Phi(\beta)=\left(\begin{array}{cccc}
1 & 0 & 0 & b d \\
0 & 1 & 0 & -a \\
-2 b & 2 a & 1 & -\left(a^{2}+d b^{2}\right) \\
0 & 0 & 0 & 1
\end{array}\right)=E\left(t, a d^{-1} s-b r\right) .
$$

Also $\Phi\left(\beta^{t}\right)=E\left(u,-a d^{-1} s-b r\right)$. Since $S L(2, \mathbf{Q}(\sqrt{ }-d))$ is generated by all $\beta, \beta^{t}$ it follows that

$$
\Phi\left(S L(2, \mathbf{Q}(\sqrt{ }-d)) \subset O^{\prime}(V) .\right.
$$

If $H_{t}$ and $H_{u}$ denote the subgroups generated by all $E(t, v)$ and $E(u, v)$ respectively, it is clear that $H_{t}$ and $H_{u}$ lie in $\Phi(S L(2, \mathbf{Q}(\sqrt{ }-d)))$. Now suppose $x$ is such that $n(x)=0$ and $x$ does not lie in the subspace spanned by $t$ or in the subspace spanned by $u$. Let $x=a t+b u+v$ where $v \in\langle t, u\rangle^{\perp}$. Then $0=n(x)=d a b+n(v)$ so that 
$n(v)>0$. But then $\tau=E\left(t,(b d)^{-1} v\right)$ maps $x$ to $b u$. Thus for any suitable $w$, $\tau E(x, w) \tau^{-1} \in H_{u}$ and $E(x, w) \in\left\langle H_{t}, H_{u}\right\rangle$. It then follows that

$$
\Phi\left(S L(2, \mathbf{Q}(\sqrt{ }-d))=O^{\prime}(V) .\right.
$$

Note that the kernel of $\Phi$ in this case is $\pm I$.

Now let $\beta=\left(\begin{array}{ll}a & 0 \\ 0 & 1\end{array}\right)$ with $a \in \mathbf{Q}^{*}$. Then $\Phi(\beta)$ fixes $r, s$ and maps $t \rightarrow a t, u \rightarrow a^{-1} u$. Thus $\Phi(\beta) \in S O(V)$ and $\theta(\Phi(\beta))=a \mathbf{Q}^{* 2}[10]$. It thus follows that the image of $A_{\mathbf{Q}}^{*}$ under $\Phi$ lies in $S O(V)$, and that the kernel consists of $\left(\begin{array}{ll}a & 0 \\ 0 & a\end{array}\right)$ with $a \in \mathbf{Q}^{*}$. It then follows that $\Phi$ maps $A_{\mathbf{Q}}^{*}$ onto $S O(V)$ and for $\beta \in A_{\mathbf{Q}}^{*}$, the spinor norm of $\Phi(\beta)$ is $\operatorname{det}(\beta) \mathbf{Q}^{* 2}$.

To obtain the image of $S L\left(2, O_{d}\right)$ under $\Phi$, we first consider the extended Bianchi group $B_{d}$ which is defined to be the maximal discrete subgroup of $P S L(2, \mathbf{C})$ which contains the Bianchi group $P S L\left(2, O_{d}\right)$. Maximal discrete subgroups of $P S L(2, \mathbf{C})$ in the commensurability class of any arithmetic Kleinian group have been described $[1]$.

In the cases here, $B_{d}$ can be described as follows. Let $\mathcal{O}=M\left(2, O_{d}\right)$ and define

$$
\operatorname{Norm}(\mathcal{O})=\left\{x \in A^{*} \mid x \mathcal{O}=\mathcal{O} x\right\} \text {. }
$$

Then $B_{d}=P(\operatorname{Norm}(\mathcal{O}))$ where $P$ denotes the natural projection $G L(2, \mathbf{C}) \rightarrow$ $P G L(2, \mathbf{C})$. If $x \in \operatorname{Norm}(\mathcal{O})$ then the ideal $(n(x))=J^{2}$ for some ideal $J$ of $O_{d}$. The mapping $x \rightarrow J$ from $\operatorname{Norm}(\mathcal{O})$ to the group of fractional ideals then induces an isomorphism

$$
\frac{B_{d}}{P G L\left(2, O_{d}\right)} \cong C_{2}
$$

where, if $C$ is the class group of $O_{d}$, then $C_{2}$ is the subgroup of all elements of order at most 2 [1]. Now $C_{2} \cong \mathbf{Z}_{2}^{t-1}$ where $t$ is the number of distinct prime divisors $p_{i}$ of $\Delta$, the discriminant of $\mathbf{Q}(\sqrt{ }-d)$, and is generated by the classes of $\mathcal{P}_{1}, \cdots, \mathcal{P}_{t}$ where $p_{i} O_{d}=\mathcal{P}_{i}^{2}$.

For each $p_{i} \mid d$, let $-d=p_{i} q_{i}$ and choose $a_{i}, b_{i} \in \mathbf{Z}$ such that $a_{i} q_{i}-b_{i} p_{i}=1$. Then

$$
\sigma_{p_{i}}=\left(\begin{array}{cc}
\sqrt{ }-d & p_{i} \\
b_{i} p_{i} & a_{i} \sqrt{ }-d
\end{array}\right) \in \operatorname{Norm}(\mathcal{O})
$$

If, in addition in the case $d \equiv 1(\bmod 4)$ and $p=2$, we let $-1-d=2 q$ and choose $a, b \in \mathbf{Z}$ such that $a q-2 b=1$, then

$$
\sigma_{2}=\left(\begin{array}{cc}
1+\sqrt{ }-d & 2 \\
2 b & a(-1+\sqrt{ }-d)
\end{array}\right) \in \operatorname{Norm}(\mathcal{O}) .
$$

Then the images of the matrices $\sigma_{p_{i}}$, where $p_{i}$ are the prime divisors of $\Delta$, under $P$, together with $P G L\left(2, O_{d}\right)$ generate $B_{d}[13]$. Note that for $d=1,3, G L\left(2, O_{d}\right)$ is not a subgroup of $A_{\mathbf{Q}}^{*}$. Thus, for $d \neq 1,3$, let $B_{d}^{\prime}$ be the subgroup of $A_{\mathbf{Q}}^{*}$ generated by these matrices $\sigma_{p_{i}}$ together with $G L\left(2, O_{d}\right)$. Thus $P B_{d}^{\prime}=B_{d}$.

In the cases $d \equiv 1,2(\bmod 4)$ choose the $\mathbf{Z}$-lattice

$$
L=\mathbf{Z} r \perp \mathbf{Z} s \perp(\mathbf{Z} t+\mathbf{Z} u)
$$

while in the case $d \equiv 3(\bmod 4)$, choose

$$
L=\left(\mathbf{Z} \frac{(r-s)}{2}+\mathbf{Z} s\right) \perp(\mathbf{Z} t+\mathbf{Z} u) .
$$


From the matrix determined at (2) above, it is immediate that in the cases $d \equiv$ $1,2(\bmod 4), \Phi\left(S L\left(2, O_{d}\right)\right) \subset O(L)$, and so also

$$
\Phi\left(S L\left(2, O_{d}\right)\right) \subset O^{\prime}(L):=O(L) \cap O^{\prime}(V) .
$$

Computing $\Phi(\beta)$ with respect to the basis $\{1 / 2(r-s), s, t, u\}$ in the case $d \equiv$ $3(\bmod 4)$, shows that, in that case $\Phi\left(S L\left(2, O_{d}\right)\right) \subset O(L)$. Furthermore a direct calculation on the generators $\sigma_{p_{i}}$ shows that, for $d \neq 1,3, \Phi\left(B_{d}^{\prime}\right) \subset S O(L)$.

Furthermore, as we have seen above, the spinor norm of $\Phi\left(\sigma_{p_{i}}\right)$ is $p_{i} \mathbf{Q}^{* 2}$ so that $\left[S O(L): O^{\prime}(L)\right] \geq 2^{t+1}$. Now locally, $L_{p}$ is unimodular for all primes $p$ where $(p, 2 d)=1$. Since the local group $O\left(L_{p}\right)$ over the $p$-adic integers is generated by symmetries about primitive vectors in $L_{p}$ of unit length, for these $p$, the spinor norm of any element of $S O(L)$ is locally a unit. Thus

$$
\left[S O(L): O^{\prime}(L)\right]=2^{t+1} .
$$

We note that $\Phi\left(\begin{array}{cc}\sqrt{ }-d & 0 \\ 0 & \sqrt{ }-d\end{array}\right)=-I$ while $P\left(\begin{array}{cc}\sqrt{ }-d & 0 \\ 0 & \sqrt{ }-d\end{array}\right)$ is the identity in $P S L(2, \mathbf{C})$. Furthermore $\left(\begin{array}{cc}\sqrt{ }-d & 0 \\ 0 & \sqrt{ }-d\end{array}\right) \in B_{d}^{\prime}$. Thus from $\Phi$ we obtain a commutative diagram, with $\Phi^{*}$ a monomorphism

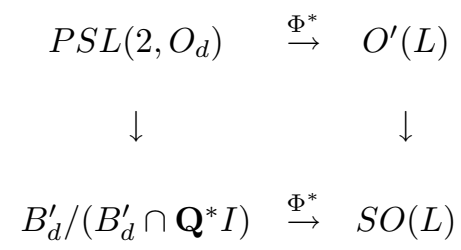

and the indexes in both cases are $2^{t+1}$. Thus $\Phi^{*}$ induces a monomorphism $B_{d} \rightarrow$ $P S O(L)$.

Now if the coefficients in $A$ are extended to $\mathbf{C}$ by tensoring, to obtain $\hat{A} \cong$ $M(2, \mathbf{C})$, then the mapping $\Phi$ can also be extended to $\hat{A}_{\mathbf{R}}^{*}$ with image in $O(V, \mathbf{R})$. The diagonal matrices in $\hat{A}_{\mathbf{R}}^{*}$ map to $\pm I$ and the images of the elements in $S L(2, \mathbf{C})$ lie in $S O(V, \mathbf{R})$. Thus we obtain a monomorphism

$$
P\left(\hat{A}_{\mathbf{R}}^{*}\right)=P S L(2, \mathbf{C}) \rightarrow P S O(V, \mathbf{R}) .
$$

As the groups $P S L(2, \mathbf{C})$ and $P S O(V, \mathbf{R})$ are isomorphic, this continuous map will then be an isomorphism. But as $B_{d}$ is a maximal discrete subgroup of $\operatorname{PSL}(2, \mathbf{C})$ and $P S O(L)$ is discrete in $P S O(V, \mathbf{R})$, it follows that this isomorphism maps $B_{d}$ onto $P S O(L)$. This then implies that the image of $P S L\left(2, O_{d}\right)$ under $\Phi^{*}$ is $O^{\prime}(L)$ in all cases except $d=1,3$.

In the cases $d=1,3$, we note that not all elements of $G L\left(2, O_{d}\right)$ lie in $A_{\mathbf{Q}}^{*}$. We can deal with these two cases by slightly altering our definitions so that

$$
A_{\mathbf{Q}}^{*}=\left\{\beta \in A^{*} \| \operatorname{det}(\beta) \mid \in \mathbf{Q}^{*}\right\}
$$

and

$$
\phi_{\beta}(v)=|\operatorname{det}(\beta)|^{-1} \beta v \tau(\beta) .
$$

It follows that $\Phi\left(A_{\mathbf{Q}}^{*}\right) \subset O(V)$ and $\Phi$ agrees with the earlier definition on elements of norm 1. Thus $\Phi\left(S L\left(2, O_{d}\right)\right) \subset O^{\prime}(L)$. The kernel of $\Phi$ restricted to $G L\left(2, O_{1}\right)$ is generated by $i I$ and in the case of $G L\left(2, O_{3}\right)$ is generated by $\exp (\pi i / 3) I$. One 
easily checks that in these cases, $-I$ does not belong to the image of $G L\left(2, O_{d}\right)$ under $\Phi$ and that $\Phi\left(G L\left(2, O_{d}\right)\right) \subset S O(L)$. Thus $\Phi$ induces a monomorphism

$$
P G L\left(2, O_{d}\right) \rightarrow S O(L)
$$

and an isomorphism carrying $P G L\left(2, O_{d}\right)$ to $P S O(L)$ and $P S L\left(2, O_{d}\right)$ to $O^{\prime}(L)$.

We summarize these results as

Theorem 2.1. For all square-free positive integers $d$, the extended Bianchi group $B_{d}$ is isomorphic to the group $P S O(L)$ where $L$ is the lattice defined at (4) and (5) above in the $\mathbf{Q}$-quadratic space $V$ at (1). The isomorphism maps $P S L\left(2, O_{d}\right)$ onto $O^{\prime}(L)$, the spinorial kernel of $S O(L)$.

\section{FUChSIAN SUBgROUPS}

The Bianchi groups $P S L\left(2, O_{d}\right)$ have been shown to be isomorphic, via $\Phi^{*}$, to the groups $O^{\prime}(L)$ where $L$ is a $\mathbf{Z}$-lattice in the quadratic space $V$. In this section, we use the isomorphism $\Phi^{*}$ to pass from maximal Fuchsian subgroups of $P S L\left(2, O_{d}\right)$ to maximal Fuchsian subgroups of $O^{\prime}(L)$ and hence to primitive representations of integers by $L$. The methods are as in [8], where the equivalence was established for the case $d=1$.

All Fuchsian subgroups considered are non-elementary so that they are not cyclic or dihedral. For a circle or straight line $\mathcal{C}$ in $\mathbf{C}$, let $\hat{\mathcal{C}}$ denote the hyperbolic plane in the upper half 3-space model of $\mathbf{H}^{3}$ represented by the hemisphere or plane in $\mathbf{H}^{3}$ orthogonal to $\mathbf{C}$ and meeting $\mathbf{C}$ in $\mathcal{C}$. Every maximal Fuchsian subgroup $F$ of $P S L\left(2, O_{d}\right)$ has the form

$$
F=\operatorname{Stab}\left(\mathcal{C}, P S L\left(2, O_{d}\right)\right)
$$

where it is understood that the elements of the stabiliser leave $\mathcal{C}$ invariant and preserve the components of $\mathbf{C} \backslash \mathcal{C}$ so that they act in an orientation-preserving way on $\hat{\mathcal{C}}$. Any such $\mathcal{C}$ with a non-elementary stabiliser has an equation of the form

$$
a|z|^{2}+B z+\bar{B} \bar{z}+c=0
$$

where $a, c \in \mathbf{Z}$ and $B=b_{1}+b_{2} \omega \in O_{d}$ where $\omega=\sqrt{ }-d$ for $d \equiv 1,2(\bmod 4)$ and $\omega=1 / 2(1+\sqrt{ }-d)$ for $d \equiv 3(\bmod 4)$. Furthermore we can assume that this representation is primitive in the sense that the g.c.d $\left(a, b_{1}, b_{2}, c\right)=1$. Up to conjugacy in $P S L\left(2, O_{d}\right)$ there is no loss of generality in assuming that $a \neq 0$. Also the Discriminant of $\mathcal{C}, D=D(\mathcal{C}):=B \bar{B}-a c$ is a conjugacy invariant (for all this see [8]). Note that $D / a^{2}$ is the square of the Euclidean radius of $\mathcal{C}$ so that $D$ is a positive integer.

On the other hand, for any positive integer $D$, let $H$ denote the quaternion algebra $\left(\frac{-d, D}{\mathbf{Q}}\right)$. With respect to the standard basis of $H$,

$$
i_{H} \rightarrow\left(\begin{array}{cc}
\sqrt{ }-d & 0 \\
0 & -\sqrt{ }-d
\end{array}\right), \quad j_{H} \rightarrow\left(\begin{array}{cc}
0 & D \\
1 & 0
\end{array}\right)
$$

induces an embedding of $H$ into $A$. If the order $\mathbf{Z}\left[1, i_{H}, j_{H}, i_{H} j_{H}\right]$ is denoted by $\Omega$, then $P\left(\Omega^{1}\right) \subset P S L\left(2, O_{d}\right)$ as an arithmetic Fuchsian subgroup, necessarily nonelementary. Furthermore, $P\left(\Omega^{1}\right)$ stabilises $\mathcal{C}_{D}$ where

$$
\mathcal{C}_{D}=\left\{\left.z \in \mathbf{C}|| z\right|^{2}=D\right\} .
$$


Thus for every positive integer $D$, there is a circle $\mathcal{C}$ corresponding to a maximal Fuchsian subgroup of $P S L\left(2, O_{d}\right)$ with $D(\mathcal{C})=D$.

The projective image of the cone $\{x \in V \otimes \mathbf{R} \mid n(x)<0\}$ is a model of hyperbolic 3-space on which $P S O(V, \mathbf{R})$ acts as the full group of orientation-preserving isometries. A hyperbolic plane in this model is the image of a 3-dimensional linear subspace of $V \otimes \mathbf{R}$, whose normal vector $w_{0}$ will be such that $n\left(w_{0}\right)>0$. Thus a maximal non-elementary Fuchsian subgroup of $O^{\prime}(L) \subset P S O(V, \mathbf{R})$ will have the form

$$
\operatorname{Stab}\left(w_{0}, O^{\prime}(L)\right)=\left\{T \in O^{\prime}(L) \mid T\left(w_{0}\right)=w_{0}\right\}
$$

for some $w_{0}$. The subspace $\left\langle w_{0}\right\rangle$ is uniquely determined whenever the stabiliser is assumed to be non-elementary. Clearly

$$
\Phi^{*}\left(\operatorname{Stab}\left(\mathcal{C}, P S L\left(2, O_{d}\right)\right)\right)=\operatorname{Stab}\left(w_{0}, O^{\prime}(L)\right)
$$

for some $w_{0}$. We now determine $w_{0}$ in terms of $\mathcal{C}$.

Note that $\Phi^{*}\left(P\left(\Omega^{1}\right)\right) \subset \operatorname{Stab}\left(w_{0}, O^{\prime}(L)\right)$ where $w_{0}=D t+u$ (see [8]). Thus

$$
\Phi^{*}\left(\operatorname{Stab}\left(\mathcal{C}_{D}, P S L\left(2, O_{d}\right)\right)\right)=\operatorname{Stab}\left(w_{0}, O^{\prime}(L)\right)
$$

and $w_{0}$ gives a primitive representation of $D d$ by $L$. Let $\mathcal{C}$ be any circle with nonelementary stabiliser in $P S L\left(2, O_{d}\right)$ and $D(\mathcal{C})=D$. Let $\mathcal{C}$ be as given at (6), and let $S=\left(\begin{array}{cc}a & \bar{B} \\ 0 & 1\end{array}\right) \in A_{\mathbf{Q}}^{*}$. Then $P S C=\mathcal{C}_{D}$. Now $S$ lies in the commensurator of $S L\left(2, O_{d}\right)$ and so $\Phi(S)$ lies in the commensurator of $O^{\prime}(L)$. Thus $\Phi^{*}\left(\operatorname{Stab}\left(\mathcal{C}, P S L\left(2, O_{d}\right)\right)\right)$ will be commensurable with $\operatorname{Stab}\left(\Phi\left(S^{-1}\right)\left(w_{0}\right), O^{\prime}(L)\right)$ and so by the remarks above, these two will be equal. Now, letting $w_{0}^{\prime}=\Phi\left(S^{-1}\right)\left(w_{0}\right)$

$$
w_{0}^{\prime}= \begin{cases}d b_{2} r+b_{1} s-c t+a u & d \equiv 1,2(\bmod 4) \\ b_{2} d\left(\frac{r-s}{2}\right)+\left(b_{1}+\left(\frac{1+d}{2}\right) b_{2}\right) s-c t+a u & d \equiv 3(\bmod 4)\end{cases}
$$

and $n\left(w_{0}^{\prime}\right)=d D$. Thus $w_{0}^{\prime} \in L$ and if, with respect to the appropriate basis, $w_{0}^{\prime}$ has coefficients $\left(x_{1}, x_{2}, x_{3}, x_{4}\right)$, then $x_{1} \equiv 0(\bmod d)$ and $\left(x_{1} / d, x_{2}, x_{3}, x_{4}\right)$ is primitive.

Notice that, if $w_{0}^{\prime} \in L$ is such that $n\left(w_{0}^{\prime}\right)=d D$, then necessarily $x_{1} \equiv 0(\bmod d)$. Thus let $L(D)$ be the set of representations of $d D$ by $L$ such that $\left(x_{1} / d, x_{2}, x_{3}, x_{4}\right)$ is primitive. For each $x \in L(D)$ we can recover the coefficients on the right hand side of (7) and hence the circle $\mathcal{C}$ with non-empty stabiliser as defined at (6).

Finally, note that the vectors $w_{0}^{\prime}$ can be replaced by their negatives. Let $\hat{O}^{\prime}(L)=$ $\left\langle O^{\prime}(L),-I\right\rangle$. Thus

Theorem 3.1. The number of conjugacy classes of non-elementary maximal Fuchsian subgroups of $P S L\left(2, O_{d}\right)$ which have discriminant $D$ is equal to the number of $\hat{O}^{\prime}(L)$ equivalence classes of representations of $d D$ from $L(D)$.

\section{ORBits Under $O^{\prime}(L)$}

To classify the maximal non-elementary Fuchsian subgroups of the Bianchi groups it is now necessary to study the orbits of $\hat{O}^{\prime}(L)$ acting on a class of elements in $L$. In this section, the global question is reduced to local ones through strong approximation in the group $O^{\prime}(L)$. Let

$$
L^{\prime}=\{x \in L \mid n(x) \in d \mathbf{Z}\} .
$$

Then $L^{\prime}=\mathbf{Z} d r \perp \mathbf{Z} s \perp(\mathbf{Z} t+\mathbf{Z} u)$ when $d \equiv 1,2(\bmod 4)$ and when $d \equiv 3(\bmod 4)$ simply replace $r$ by $(r-s) / 2$. Now put

$$
M=\left\{x \in L \mid f\left(x, L^{\prime}\right) \subset 2 d \mathbf{Z}\right\} .
$$


Then $L^{\prime}$ and $M$ are sublattices of $L$ invariant under $O(L)$, with $M=\mathbf{Z} r \perp \mathbf{Z} s \perp(\mathbf{Z} 2 t+$ $\mathbf{Z} 2 u)$ when $d \equiv 1,2(\bmod 4)$, and $M=2 L$ when $d \equiv 3(\bmod 4)$. Define

$$
L(D)=\left\{x \in L^{\prime} \mid n(x)=d D \text { and } f(x, M)=2 d \mathbf{Z}\right\}
$$

so that $L(D)$ is as described in Theorem 3.1.

The group $O^{\prime}(L)$ acts on $L(D)$ and we let $S(L, D)$ denote the collection of spinor equivalence classes of $L(D)$ under this action. Set

$$
N(L, D)=|S(L, D)| \text {. }
$$

In the same way, if $L_{p}$ denotes the localisation of $L$ at $p$, then $N\left(L_{p}, D\right)$ will denote the number of spinor equivalence classes of $L_{p}(D)$ under the action of $O^{\prime}\left(L_{p}\right)$.

Theorem 4.1.

$$
N(L, D)=\prod_{p \mid 2 d} N\left(L_{p}, D\right) .
$$

Let $\Gamma: S(L, D) \rightarrow \prod_{p \mid 2 d} S\left(L_{p}, D\right)$ be the natural map from $S(L, D)$ to the cartesian product of the sets $S\left(L_{p}, D\right)$ over $p \mid 2 d$. The theorem follows by proving that $\Gamma$ is a bijection using arguments similar to those for Theorems 2.1 and 2.3 of $[6]$.

In the proof of this theorem given below and in the local counting theorems in the next section, we rely on results and concepts in the theory of quadratic forms which are to be found in [10] whose notation we also adopt. For convenience, we now outline some of these concepts.

As above $L$ will denote a lattice over a ring $R$ with field of quotients $F$ where $R$ will be either $\mathbf{Z}$ or $\mathbf{Z}_{p}$. We continue to use $n$ and $f$ for the quadratic maps and associated bilinear forms respectively. The scale of $L, \mathbf{s}(L)$, is the $R$-module generated by $f(L, L)$ and the norm of $L, \mathbf{n}(L)$, is the $R$-module generated by $n(L)$. If $L$ has rank $r$ and $\left\{x_{1}, x_{2}, \ldots, x_{r}\right\}$ is a free $R$-basis, then the volume of $L, \mathbf{v}(L)$, is the $R$-module generated by the discriminant of the basis $=\operatorname{det}\left[f\left(x_{i}, x_{j}\right)\right]$. In all cases, $\mathbf{v}(L) \subset \mathbf{s}(L)^{r}$.

Definition 4.2. A lattice is $\mathcal{A}$-modular, where $\mathcal{A}$ is a fractional ideal, if $\mathbf{s}(L)=\mathcal{A}$ and $\mathbf{v}(L)=\mathcal{A}^{r}$. If $\mathcal{A}=R$, then it is unimodular.

(See $[10, \S 92]$.

Over a local ring, $L$ splits into one and two dimensional modular components, which when grouped together appropriately, give a splitting

$$
L=L_{1} \perp L_{2} \perp \ldots \perp L_{t}
$$

into modular components such that $\mathbf{s}\left(L_{t}\right) \subset \mathbf{s}\left(L_{t-1}\right) \subset \ldots \subset \mathbf{s}\left(L_{1}\right)$. This is a Jordan splitting and the ranks, scales and norms are unique for $L$. (See $[10, \S 91]$ in particular for the precise statement on uniqueness.)

With respect to a basis $\left\{x_{1}, x_{2}, \ldots, x_{r}\right\}$ of $V_{p}$ over $\mathbf{Q}_{p}$, each $\sigma_{p} \in O\left(V_{p}\right)$ gives $\sigma_{p}\left(x_{i}\right)=\sum a_{i j} x_{j}$. A (topological) norm can be defined locally on $\sigma_{p}$ by

$$
\left\|\sigma_{p}\right\|_{p}=\max \left|a_{i j}\right|_{p} \text {. }
$$

Choosing a basis of $V$ over $\mathbf{Q}$, and using this same basis locally, each $\sigma \in O(V)$ will inherit a local norm. Clearly, for all but a finite number of $p,\|\sigma\|_{p}=1$. Also, if $\|\sigma\|_{p}=1$ for all $p$, then $\sigma \in O(L)$ where $L$ is the $\mathbf{Z}$-lattice with free basis $\left\{x_{1}, x_{2}, \ldots, x_{r}\right\}[10, \S 101]$.

We now turn to the proof of Theorem 4.1 and first establish 
A. The injectivity of $\Gamma$.

Thus let $x, y \in L(D)$ be such that they are locally spinor equivalent at each $p \mid 2 d$. We first show that they are also locally spinor equivalent at each $p$ with $(p, 2 d)=1$. If $(p, 2 d)=1$, then $L_{p}^{\prime}=M_{p}=L_{p}$ and $L_{p}$ is unimodular. Since $x, y$ are such that $f\left(x, L_{p}\right)=\mathbf{Z}_{p}=f\left(y, L_{p}\right), x, y$ are primitive in $L_{p}$. But $O\left(L_{p}\right)$ acts transitively on primitive vectors of the same length (e.g. [5]), so there exists $\phi_{p} \in O\left(L_{p}\right)$ with $\phi_{p}(x)=y$. Now the orthogonal complement of $y$ in $L_{p}$ will be split by a rank 2 unimodular component. Over a non-dyadic field, this component admits isometries of all possible spinor norms [10, §92]. Extending this to $L_{p}$ means that $\phi_{p}$ can be adjusted to lie in $O^{\prime}\left(L_{p}\right)$.

Thus for all primes $p$, we have $\phi_{p} \in O^{\prime}\left(L_{p}\right)$ such that $\phi_{p}(x)=y$. By Witt's Theorem, there exists $\tau \in O(V)$ such that $\tau(x)=y$ and we can again adjust such that $\tau \in O^{\prime}(V)[10, \S 101: 8]$. Then $\tau_{p}^{-1} \phi_{p}(x)=x$ for each prime $p$. Let $V=\mathbf{Q} x \perp W$ so that $W$ is a 3 -dimensional indefinite subspace of $V$, and $\tau_{p}^{-1} \phi_{p} \in O^{\prime}\left(W_{p}\right)$. Let $S$ be the set of all primes and let $T$ the finite subset of those such that $\left\|\tau_{p}\right\|_{p} \neq 1$. Then, by the strong approximation theorem [10, $1104: 4]$, there exists $\psi \in O^{\prime}(W)$ such that $\left\|\psi_{p}-\tau_{p}^{-1} \phi_{p}\right\|_{p}<\epsilon$ for $p \in T$ and $\left\|\psi_{p}\right\|_{p}=1$ for all other $p$. Now extend $\psi$ by the identity to $O^{\prime}(V)$ and set $\sigma=\tau \psi$. Then $\sigma(x)=y$ and $\left\|\sigma_{p}\right\|_{p}=1$ for all $p$, provided $\epsilon$ was chosen sufficiently small initially. Thus $\sigma \in O^{\prime}(L)$ and $\Gamma$ is injective.

B. The surjectivity of $\Gamma$.

Let $x_{p} \in L_{p}(D)$ be given for each $p \mid 2 d$. For the remaining primes $L_{p}$ is unimodular and, since these primes are odd, there will exist $x_{p} \in L_{p}$ with $n\left(x_{p}\right)=$ $d D$ and $f\left(x_{p}, L_{p}\right)=\mathbf{Z}_{p}$. By the Hasse-Minkowski Thorem, there now exists $z \in V$ with $n(z)=d D$. Let $U$ be the finite set of primes where either $p \mid 2 d$ or $f\left(z, L_{p}\right) \neq$ $\mathbf{Z}_{p}$. By Witt's Theorem, there exists $\tau_{p} \in O\left(V_{p}\right)$ such that $\tau_{p}\left(x_{p}\right)=z$ for each $p \in U$ and for the remaining $p$ we can choose $\tau_{p} \in O\left(L_{p}\right)$ (e.g. [5]). Since $d D>0$ and $V$ is 4-dimensional, we can adjust locally as before such that $\tau_{p} \in O^{\prime}\left(V_{p}\right)$. Again by the strong approximation theorem, there exists $\psi \in O^{\prime}(V)$ such that $\left\|\psi_{p}-\tau_{p}^{-1}\right\|_{p}<\epsilon$ for all $p \in U$ and $\left\|\psi_{p}\right\|_{p}=1$ for the remainder. Let $y=\psi(z)$, so that $n(y)=d D$. Then $y$ is locally close to $x_{p}$ and so $y \in L_{p}(D)$ for $p \in U$. For the remaining primes, $\psi_{p} \in O^{\prime}\left(L_{p}\right)$ and so $y \in L_{p}(D)$. Thus $y \in L(D)$. Moreover, $\psi_{p} \tau_{p} \in O^{\prime}\left(L_{p}\right)$ and $\psi_{p} \tau_{p}\left(x_{p}\right)=y$ for all $p \mid 2 d$. Hence, the class of $y$ in $S(L, D)$ is mapped by $\Gamma$ onto the cartesian product over $p \mid 2 d$ of the classes of $x_{p}$. Thus $\Gamma$ is surjective.

\section{Local Calculations}

In this section, we calculate the local orbit sizes $N\left(L_{p}, D\right)$ which appear in Theorem 4.1 and study the effect of $-I$ on the $O^{\prime}(L)$ orbits. In this way, we obtain the number of conjugacy classes of maximal non-elementary Fuchsian subgroups of $P S L\left(2, O_{d}\right)$ with discriminant $D$.

In the arguments that follow, some general results on isometries are used together with specifically constructed isometries. We first establish our notation for these. Here $L$ is either a lattice over $\mathbf{Z}$ or over $\mathbf{Z}_{p}$, but the symbols $r, s, t, u$ are used for the vectors described above. For $z \in L$ such that $n(z) \neq 0$, let $\Psi(z)$ denote the symmetry about $z$. The local groups $O\left(L_{p}\right)$ are generated by integral symmetries when $p$ is odd $[10, \S 92]$. Note that $\Psi(t+u) \Psi(s) \in O^{\prime}(L)$ and interchanges $t$ and $-u$. For $\alpha \in \mathbf{Z}_{p}$, let $T(\alpha)$ denote the isometry sending $t$ to $\alpha t$ and $u$ to $\alpha^{-1} u$, so that $T(\alpha)$ has local spinor norm $\alpha \mathbf{Q}_{p}^{* 2}$. For the Eichler transformations $E(t, v)$ 
and $E(u, v)$ defined at (3) to be integral and hence in $O^{\prime}\left(L_{p}\right)$, we require that $f\left(v, L_{p}\right) \subset \mathbf{Z}_{p}$ and $d n(v) \in \mathbf{Z}_{p}$.

Theorem 5.1. Let $p$ be odd, $p \mid d$.

1. If $(p, D)=1$, then $N\left(L_{p}, D\right)=1$.

2. If $p \mid D$, then $N\left(L_{p}, D\right)=4$ if and only if $\left(\frac{d^{-1} D}{p}\right)=1$; otherwise $N\left(L_{p}, D\right)$ $=2$.

Proof. If $x \in L_{p}(D)$, then $x=d b_{2} r+b_{1} s+c t+a u$ where $n(x)=d D$ and $\left(a, b_{1}, b_{2}, c\right)=1$. Thus either $x$ is primitive in $L_{p}$ or $b_{2}$ is a unit and $p$ divides $a, b_{1}, c$. The second case implies that $d b_{2}^{2} \equiv D\left(\bmod p^{2}\right)$ so that $\left(\frac{d^{-1} D}{p}\right)=1$.

1. Since $(p, D)=1, x$ is primitive in $L_{p}$. Now $L_{p}$ has a Jordan splitting with a rank 1 unimodular component and a rank $3 p$-modular component. The $p$-modular sublattice $\mathbf{Z}_{p} x$ splits $L_{p}$ with orthogonal complement split by a rank $2 p$-modular component by the uniqueness of Jordan splittings [10, §91]. Since this rank $2 p$ modular component has isometries of all possible spinor norms [10, §92:5], and $O\left(L_{p}\right)$ acts transitively on primitive vectors of the same length, the result follows.

2. If $c$ is a unit in $\mathbf{Z}_{p}$, the isometry $E\left(u, c^{-1}\left(b_{2} r+b_{1} d^{-1} s\right)\right) \in O^{\prime}\left(L_{p}\right)$ and maps $x$ to $c t+c^{-1} D u$. Since we can interchange $t$ and $-u$, the same result holds if $a$ is a unit. Let $e$ be a fixed non-square in $\mathbf{Z}_{p}$. Then using elements in $O^{\prime}\left(L_{p}\right)$ of one of the forms $T\left(c^{-1}\right)$ or $T\left(c^{-1} e\right)$, it follows that $x$ is spinor equivalent to $t+D u$ or $e t+e^{-1} D u$. If neither $a$ nor $c$ is a unit, it follows that $x$ is not primitive and so $\left(\frac{d^{-1} D}{p}\right)=1$. We return to this case below, but show first of all, that the two representations just obtained are not spinor equivalent.

Suppose $\phi \in O^{\prime}\left(L_{p}\right)$ maps $t+D u$ to $e t+e^{-1} D u$. Then $\tau=T\left(e^{-1}\right) \phi$ fixes $t+D u$ and has spinor norm $e \mathbf{Q}_{p}^{* 2}$. Then $\tau(r)$ has the form $a_{1} r+b s+c_{1}(t-D u)$ where $a_{1}^{2} \equiv 1(\bmod p)$. If $a_{1} \equiv-1(\bmod p)$, let $\psi=\Psi(\tau(r)-r) \tau$ and if $a_{1} \equiv 1(\bmod p)$, let $\psi=\Psi(r) \Psi(\tau(r)+r) \tau$. Note that the norms of $\tau(r) \pm r$ in the appropriate cases are squares in $\mathbf{Q}_{p}^{*}$. Now $\psi \in O\left(L_{p}\right)$ fixes $r$ and $t+D u$. Thus $\psi(s)$ has the form $a_{2} s+c_{2}(t-D u)$ with $a_{2}^{2} \equiv 1(\bmod p)$. As before, define the elements $\Psi(\psi(s)-s) \psi$ or $\Psi(s) \Psi(\psi(s)+s) \psi$ so that they fix $r, s, t+D u$ and so must be the identity. In particular, they must have determinant 1 and trivial spinor norm. In each of the four cases we obtain a contradiction to this.

We now return to the case where $x$ is not primitive and $b_{2}$ is a unit. By Hensel's lemma, there exists $b \in \mathbf{Z}_{p}$ such that $c d^{-1}+2 b_{2} b-a b^{2}=1$. The coefficient of $t$ in $E(t, b r)(x)$ is now $d$. The $s$ term can be removed by using $E\left(u, b_{1} d^{-2} s\right)$ so that $x$ is spinor equivalent to $d y$ with $y=e_{1} r+t+d_{1} u$ where $e_{1}$ is a unit and $d^{-1} D \equiv e_{1}^{2}(\bmod p)$. Moreover, using transformations $E(u, \lambda r)$, we can reduce $e_{1}$ to two choices. It remains to show that $y$ and $y^{\prime}=-e_{1} r+t+d_{1} u$ are not spinor equivalent. Assume that $\phi \in O^{\prime}\left(L_{p}\right)$ sends $y$ to $y^{\prime}$. Then $\Psi(r) \phi$ fixes $y$. Since the orthogonal complement of $y$ is $p$-modular and splits $L_{p}, \Psi(r) \phi$ must be a product of an odd number of integral symmetries on this complement. But then $\phi$ is not in $O^{\prime}\left(L_{p}\right)$. Hence $N\left(L_{p}, D\right)=4$.

Corollary 5.2. With notation as in the above proof, et $+e^{-1} D u$ is equivalent to $t+D u$ under the action of $-I$ if and only if $p \equiv 3(\bmod 4)$. Also dy is equivalent to $d y^{\prime}$ under $-I$ for all relevant $p$. 
Proof. The first part follows since $-t-D u$ is spinor equivalent to $e t+e^{-1} D u$ if and only if $p \equiv 3(\bmod 4)$. For the cases where $N\left(L_{p}, D\right)=4, T(-1)\left(y^{\prime}\right)=-y$ and there exists $\phi \in O\left(L_{p}\right)$ fixing $y$ with spinor norm $-\mathbf{Q}_{p}^{* 2}$ since the orthogonal complement of $y$ is a rank three $p$-modular lattice splitting $L_{p}$.

Theorem 5.3. Let $d$ be odd.

1. $N\left(L_{2}, D\right)=1$ when $d \equiv 3(\bmod 4)$.

2. $N\left(L_{2}, D\right)=1$ when $d \equiv 1(\bmod 4)$, and $D \equiv 3,4,7(\bmod 8)$.

3. $N\left(L_{2}, D\right)=2$ when $d \equiv 1(\bmod 4)$, and $D \equiv 0,2,6(\bmod 8)$.

4. $N\left(L_{2}, D\right)=3$ when $d \equiv D \equiv 1(\bmod 4)$.

Proof. Since $d$ is odd, all $x \in L_{2}(D)$ are primitive in $L_{2}$. Let $x=c t+a u+w$ where $w \in\langle t, u\rangle^{\perp}$. If $a$ or $c$ is a 2-adic unit, we can use Eichler transformations to obtain that $x$ is spinor equivalent to $c t+c^{-1} D u$. Now $T\left(c^{-1}\right)$ maps this to $t+D u$.

When $d \equiv 3(\bmod 4),\langle t, u\rangle^{\perp}$ is a binary even unimodular lattice, so that we can adjust so that $x$ is always spinor equivalent to $t+D u$. Also in this case, if neither $a$ nor $c$ is a unit, via Eichler transformations $x$ is spinor equivalent to a vector whose $t$ coefficient is a unit. Thus 1 follows.

Now suppose that $d \equiv 1(\bmod 4)$ and $w=d b_{2} r+b_{1} s$. As above, if $a$ or $c$ is a unit, then $x$ is spinor equivalent to $c t+c^{-1} D u, c$ a unit. If $D \not \equiv 0(\bmod 4)$, then we can compose $T\left(c^{-1}\right)$ with an isometry of the orthogonal complement of $t+D u$ to get that $x$ is spinor equivalent to $t+D u[10, \S 91: 8, \S 93: 20]$.

When $D \equiv 0(\bmod 4)$, using $\Psi(r) \Psi(r+2 s) T(5) \in O^{\prime}\left(L_{2}\right)$ we get that $x$ is spinor equivalent to $y=t+D u$ or to $-y$.

If $D \equiv 4(\bmod 8)$, using $\Psi(r+s) \Psi(r+s+t-D u) T(e) \in O\left(L_{2}\right)$ where $e \equiv$ $-1(\bmod 4)$ shows that $y$ and $-y$ are spinor equivalent.

Next we show that when $D \equiv 0(\bmod 8), y$ and $-y$ are not spinor equivalent. Suppose they were via $\psi$ and $\phi=\psi \circ-I$. So $\phi$ fixes $y$. Let $z=t-D u$. Then since $\phi(y-z) \in 2 D L_{2}, \phi(z) \equiv z\left(\bmod 2 D L_{2}\right)$. By Hensel's lemma, there exists $\mu \in \mathbf{Z}_{2}$ such that the $s$-component of $\Psi(s+\mu z) \phi(z)$ is zero. Similarily there exists $\lambda$ such that $\tau=\Psi(r+\lambda z) \Psi(s+\mu z) \phi$ fixes both $y$ and $z$ and so lies in the orthogonal group of $J=\mathbf{Z}_{2} r+\mathbf{Z}_{2} s$. But $\theta(\tau)=-1 \notin \theta(S O(J))$ so that $y$ cannot be equivalent to $-y$.

Now assume $a$ and $c$ are both nonunits so that $D \equiv b_{1}{ }^{2}+b_{2}{ }^{2}(\bmod 4)$. This is impossible for $D \equiv 0,3(\bmod 4)$ and $N\left(L_{2}, D\right)=1$ or 2 in these cases. For $D \equiv 2(\bmod 4)$, both $b_{1}$ and $b_{2}$ must be units, and $x$ is spinor equivalent to $d r+$ $s+2 t+2 a_{1} u \in M_{2}$ (using Eichler transformations); thus $N\left(L_{2}, D\right)=2$ since $t+D u \notin M_{2}$. When $D \equiv 1(\bmod 4)$, either $b_{1}$ or $b_{2}$ is a unit, but not both. Note that $\Psi(r-s)$ interchanges these two possibilities, and that the orthogonal complement $K$ of $x$ in $L_{2}$ splits $L_{2}$. Since $\theta(S O(K))$ only has unit values modulo squares by Theorem 3.14(i) in [3] the two possibilities must lead to spinor inequivalent orbits. If $b_{1}$ is a unit $x$ is spinor equivalent to $s+2 t+2 a_{1} u \in M_{2}$, and if $b_{2}$ is a unit $x$ is equivalent to $d r+2 t+2 a_{1} u \in M_{2}$. Hence $N\left(L_{2}, D\right)=3$.

Corollary 5.4. All spinor orbits in the above theorem are fixed by $-I$ except when $d \equiv 1(\bmod 4)$ and $D \equiv 0(\bmod 8)$.

Remark. When $d=1$, the number of conjugacy classes of maximal Fuchsian subgroups of discriminant $D$ in $P S L\left(2, O_{1}\right)$ can now be computed. This was also done in [8] using Siegel's analytical results. 
Theorem 5.5. Let d be even.

1. $N\left(L_{2}, D\right)=1$ when $D \equiv 4(\bmod 8)$.

2. $N\left(L_{2}, D\right)=2$ when $D \equiv 1,3(\bmod 4)$, or $D \equiv 0,8, d+8, d+12(\bmod 16)$.

3. $N\left(L_{2}, D\right)=4$ when $D \equiv d, d+4(\bmod 16)$.

Proof. Let $x=d b_{2} r+b_{1} s+c t+a u \in L_{2}(D)$. As in the previous theorem, if $a$ or $c$ is a unit, and $D \equiv 1,2,3(\bmod 4)$, the orthogonal complement of $t+D u$ represents enough units so that $x$ is spinor equivalent to $t+D u$. In the remaining case with $D \equiv 0(\bmod 4), \Psi(r) \Psi(r+s) T(1+d) \in O^{\prime}\left(L_{2}\right)$, and $x$ is spinor equivalent to either $t+D u$ or $5 t+5^{-1} D u$. When $D \equiv 4(\bmod 8)$, the two possibilities are spinor equivalent using $\Psi(s) \Psi(s+t-D u) T(5) \in O^{\prime}\left(L_{2}\right)$. When $D \equiv 0(\bmod 8)$, modify the argument in Theorem 5.3 to show they are not spinor equivalent. As there, remove the $s$-component so that $\Psi(s+\mu z) \phi(z)=2 D b r+e z$ with $e \equiv 1(\bmod 2 D)$. Then $-d=4 D b^{2}-d e^{2}$ and hence $2 \mid b$. The $r$-component can be removed using $\Psi(r+\lambda z)$, and the result then follows since $5 \notin \theta(S O(J))$.

Now assume $a$ and $c$ are not units, but $x$ is primitive in $L_{2}$ so that $b_{1}$ is a unit. Then $d b_{2}{ }^{2}+1+a c \equiv D(\bmod 8)$ and $D$ must be odd. If $a \equiv c \equiv 2(\bmod 4)$, then $D \equiv 5,5+d(\bmod 8)$; use $E\left(t, \lambda d^{-1} s\right)$ on $x$ to change the coefficient of $t$ to 2 , and then use $E\left(u, \mu r+\nu d^{-1} s\right)$ to get $x$ spinor equivalent to $d b r+s+2 t+2 a_{1} u \in M_{2}$ with $b=0$ when $D \equiv 5(\bmod 8)$ and $b=1$ otherwise. Similarly, if $a$ or $c$ is divisible by $4, x$ is spinor equivalent to $s+2 t+4 a_{1} u \in M_{2}$ when $D \equiv 1(\bmod 8)$, and to $d r+s+2 t+4 a_{1} u \in M_{2}$ when $D \equiv 1+d(\bmod 8)$. Since $d \equiv 2(\bmod 4), D$ uniquely determines which of the four possibilities for $x$ occurs with a given $d$. None of these representations are spinor equivalent to $t+D u$ so that $N\left(L_{2}, D\right)=2$ for $D \equiv 1,3(\bmod 4)$.

It remains to consider $x=d y$ with $y \in L_{2}$ and $n(y)=D d^{-1}$ so necessarily $D \equiv 2(\bmod 4)$. Then $y$ is spinor equivalent to $r+t+\left(D d^{-1}-1\right) d^{-1} u$ when $y \notin M_{2}$ (first get the coefficient of $u$ a non-unit, and then the coefficient of $t$ can be made equal to 1$)$. If $y \in M_{2}$, then $n(y) \equiv 1+b_{1}^{2} d^{-1} \equiv 1,1+d(\bmod 8)$, so that $D \equiv d, d+4(\bmod 16)$. In this case $y$ or $-y$ is spinor equivalent to $r+b s+2 t+2 a u$, with $b=0$ when $D \equiv d(\bmod 16)$, and $b=1$ when $D \equiv d+4(\bmod 16)$. The orbits of $y$ and $-y$ are inequivalent, using Theorem 3.14(i) in [3] on the orthogonal complement of $y$.

Corollary 5.6. The two orbits for $D \equiv d, d+4(\bmod 16)$ corresponding to $y$ and $-y$ are interchanged by $-I$, as are the two orbits when $d \equiv 2(\bmod 8)$ and $D \equiv 0(\bmod 8)$. All other orbits are fixed by $-I$.

Proof. In the one non-obvious case use $\Psi(r+s) \Psi(s) T(5)$.

In order to state our results in a compact form we introduce the following notation.

$$
\begin{gathered}
m(\geq 0)=\#\left\{\text { primes } p|p \equiv 1(\bmod 4), \quad p|(d, D), \quad\left(\frac{d^{-1} D}{p}\right)=1\right\} . \\
c_{m}=\frac{1}{2}\left(1+\frac{1}{2^{m}}\right) .
\end{gathered}
$$

Theorem 5.7. Let $n_{d}(D)$ be the number of conjugacy classes of maximal nonelementary Fuchsian subgroups of discriminant D lying in $P S L\left(2, O_{d}\right)$. 
1. $n_{d}(D)=\frac{1}{2} N(L, D)$ if there exists a prime $p$ dividing $(d, D)$ with $p \equiv 3(\bmod 4)$, or if $d \equiv 1,2,5(\bmod 8)$ and $D \equiv 0(\bmod 8)$.

2. $n_{d}(D)=c_{m+1} N(L, D)$ if $d \equiv 2(\bmod 4), D \equiv d, d+4(\bmod 16)$, and there is no prime $p$ dividing $(d, D)$ with $p \equiv 3(\bmod 4)$.

3. $n_{d}(D)=c_{m} N(L, D)$ in the remaining cases.

Proof. To calculate the action of $-I$ on the global orbits $S(L, D)$, recall from Theorem 4.1 that the map from $S(L, D)$ to the cartesian product over $p \mid 2 d$ of the local orbits $S\left(L_{p}, D\right)$ is a bijection. When there exists at least one prime $p$ dividing $d$ with $p \equiv 3(\bmod 4)$, or when $d \equiv 1,2,5(\bmod 8)$ and $D \equiv 0(\bmod 8)$, then by Corollaries 5.2, 5.4 and 5.6, $-I$ does not act trivially on any $O^{\prime}(L)$ orbit, and the first part follows. In the remaining cases it suffices to consider the $p$-adic orbits with $N\left(L_{p}, D\right)=4$ and the 2-adic orbits. Assume first the 2-adic orbits are all fixed by $-I$. Locally at the $m$ odd primes where $N\left(L_{p}, D\right)=4,2^{m}$ of the orbits are fixed by $-I$, and the remaining orbits are interchanged in pairs. Hence $n_{d}(D)=c_{m} N(L, D)$. When $d \equiv D \equiv 2(\bmod 4)$, where two of the 2 -adic orbits are interchanged, the argument needs a slight modification.

Remark. A simple calculation from the results above, show that, for $d=p$ where $p$ is a prime $\equiv 3(\bmod 4)$, then $n_{d}(D)=1$ except in the cases where $D \equiv 0(\bmod p)$ and $D / p$ is a square $(\bmod p)$, in which case $n_{d}(D)=2$. These cases were also considered in [12] where it was shown that each $P G L\left(2, O_{d}\right)$ conjugacy class of maximal Fuchsian subgroups in $P S L\left(2, O_{d}\right)$ had a unique representative which was the stabiliser of a circle

$$
p|z|^{2}-\sqrt{ }(-p) m z+\sqrt{ }(-p) m \bar{z}+p c=0
$$

where $m, c \in \mathbf{Z}$ and $0 \leq m<p / 2$. When $m=0$ this circle has discriminant $D=-c$, otherwise $D=p\left(m^{2}-p c\right)$ and so we see that the numbers agree with those above. Thus, in these cases, the $\operatorname{PSL}\left(2, O_{d}\right)$ conjugacy classes must all be fixed under the action of $P G L\left(2, O_{d}\right)$. This also occurs when $d=2$, which was also considered in [12], but does not occur in the case $d=1$ as was already observed in [8]. It should be noted that these cases above give particularly simple results. By contrast, the reader is invited to calculate $n_{5}(D)$, which takes the values $1,2,3,4,6,9$ depending on the residue class of $D(\bmod 200)$.

\section{REFERENCES}

[1] A. Borel, Commensurability classes and volumes of hyperbolic 3-manifolds, Ann. Sci. Norm. Pisa 8 (1981), 1 - 33. MR 82j:22008

[2] J. Elstrodt, F. Grunewald and J. Mennicke, Discontinuous groups on three-dimensional hyperbolic space : analytical theory and arithmetic applications, Russian Math. Surveys 38 (1983), 137 - 168. MR 87c: 11053

[3] A.G. Earnest and J.S. Hsia, Spinor norms of local integral rotations II, Pacific J. Math. 61 (1975), 71 - 86. MR 53:7946

[4] B. Fine, The algebraic theory of the Bianchi groups, Marcel - Dekker (1989). MR 90h:20002

[5] D.G. James and S.M. Rosenzweig, Associated vectors in lattices over valuation rings, Amer. J. Math. 90 (1968), 295 - 307. MR 36:3754

[6] D.G. James Integral sums of squares in algebraic number fields, Amer. J. Math. 113 (1991), 129 - 146. MR 92j: 11036

[7] C. Maclachlan, Fuchsian subgroups of the groups $P S L\left(2, O_{d}\right)$, Low-Dimensional Topology and Kleinian groups, ed. D.B.A. Epstein, L.M.S. Lecture Notes Series 112 (1987), 251 258. MR 89a: 11049

[8] C. Maclachlan and A.W. Reid, Parametrizing Fuchsian Subgroups of the Bianchi Groups, Canadian J. Math. 43 (1991), 158 - 181. MR 92d:11040 
[9] C. Maclachlan and A.W. Reid, The arithmetic structure of tetrahedral groups of hyperbolic isometries, Mathematika 36 (1989), 221 - 240. MR 91b:11055

[10] O.T. O'Meara, Introduction to Quadratic Forms, Springer - Verlag (1963). MR 27:2485

[11] T. Tamagawa, On the structure of orthogonal groups, Amer. J. Math. 80 (1958), 191 - 197. MR 20:71

[12] L. Ya. Vulakh, Classification of Fuchsian subgroups of some Bianchi groups, Canadian Math. Bull. 34 (1991), 417 - 422. MR 92i:11047

[13] L. Ya. Vulakh, Maximal Fuchsian subgroups of extended Bianchi groups, Number Theory with an Emphasis on the Markoff Spectrum, ed. A. Pollington Marcel - Dekker (1993), 297 - 310. MR 94g:11028

Department of Mathematics, Pennsylvania State University, University Park, PennSYLVANIA 16802

E-mail address: james@math.psu.edu

Department of Mathematical Sciences, University of Aberdeen, Old Aberdeen, Aberdeen AB9 2TY, Scotland

E-mail address: cmac@maths.aberdeen.ac.uk 